\title{
A Joint Strategy for Fair and Efficient Energy Usage in WLANs in the Presence of Capture Effect
}

\author{
Bilal Khan ${ }^{1}$, Rana Asif Rehman ${ }^{1}\left(\mathbb{D}\right.$ and Byung-Seo Kim ${ }^{2, *}$ (i) \\ 1 Department of Computer Science, National University of Computer and Emerging Sciences, \\ Chiniot-Faisalabad Campus, Chiniot 35400, Pakistan; bilalkhan83@hotmail.com (B.K.); \\ r.asif@nu.edu.pk (R.A.R.) \\ 2 Department of Software and Communication Engineering, Hongik University, Sejong City 30016, Korea \\ * Correspondence: jsnbs@hongik.ac.kr
}

Received: 10 March 2019; Accepted: 21 March 2019; Published: 30 March 2019

\begin{abstract}
Capture effect has been shown as a physical layer (PHY) phenomenon of modern wireless devices that improves the performance of wireless local area networks (WLANs) in terms of throughput. In this paper, however, we explore the effect of PHY capture in the domain of energy efficiency. Analysis model that takes into account the effect of PHY capture is backed up by ns- 2 simulations show that capture effect improves energy efficiency of WLAN by $20 \%$. This improvement, however, results in unfairness, i.e, a group of nodes located far away from the Access Point (AP) is three times less energy efficient than the group of nodes located closer to the AP. To resolve the unfairness caused by the capture effect, furthermore, this paper proposes a joint strategy of adaptive transmission power control (ATXPR) and contention window adjustment (CWADJ). Namely, a node that suffers transmission failure due to another node capturing the channel steps up its transmission power according to the transmission power control algorithm and refrains from increasing its contention window according to contention window adjustment mechanism, respectively. Our proposed joint strategy is $99 \%$ fair while maintaining overall energy efficiency of the network.
\end{abstract}

Keywords: PSM; CAM; PHY capture; energy efficiency; energy unfairness

\section{Introduction}

With the increase in the popularity of smart phones, laptops, PDAs (Personal Digital Assistant), and other hand-held devices, wireless local area networks (WLANs) are rapidly growing and are widely deployed. These handheld devices are battery operated and need to conserve energy. It is stated that the Internet today accounts for about $2 \%$ of the total energy consumption, with further shifting of offline services online, this percentage may grow in coming years [1]. A major fraction of the energy consumption in these handheld devices comes from the WLAN interface that accounts for more than half of the total device power budget under typical use [2] and can quickly drain the phone's battery when transmitting at high peak rates. This enormous energy consumption by the wireless devices not only puts a burden on the user but also affects the environments in the form of global warming.

In order to conserve energy in WLANs, IEEE 802.11 devises a power save mode (PSM) [3]. In PSM, a node periodically wakes up to listen to a beacon frame transmitted by access point (AP) that announces the buffered frames. If there is any frame waiting for the node, then it sends a PS-POLL (Power Save Polling) to retrieve the buffered frame. Otherwise it goes back to sleep mode.

In contrast to PSM, IEEE 802.11 also operates in continuous active mode or constantly awake mode (CAM). Nodes operating in CAM use a carrier sense multiple access with collision avoidance 
(CSMA/CA)-based distributed coordinated function (DCF) to send frames without going into sleep mode. Similarly, nodes remain awake all the timse and can receive frames from the AP anytime. All clients initially choose randomly a backoff counter from the minimum contention window (CW) and starts counting down after sensing the channel idle for distributed inter-frame space (DIFS) time. A client makes a transmission attempt when its backoff counter reaches zero and all other clients hearing the transmission freeze their counters and set their network allocation vector (NAV) for the time of transmission. The transmission is successful when no other client's backoff counter reaches zero simultaneously. In this case the sender receives ACK frame after inter-frame space (IFS) duration from the destination otherwise collision happens. In case of collision, all clients involved in collision wait for the extended inter-frame space (EIFS) duration, increase their CW according to the binary exponential backoff algorithm and the clients again start contending for the channel access by decreasing the backoff counter. The same process is repeated unless the transmission is successful or the number of retries reaches predefined retry limit in which case the frame is discarded and the upper layers are notified of the transmission failure. Obviously, PSM which is specifically devised for power saving are more energy efficient than CAM as it goes to the sleep mode in case it does not have data to send or receive. However, the PSM is not suitable for delay sensitive applications, as the client that has a frame buffered at the AP has to wait for a beacon interval to retrieve it [4]. In addition, to reduce delay, some devices change its operation mode from PSM to CAM as the traffic load exceeds a certain level [5].

In this paper, we analyze the energy consumption in CAM in the presence of some real network conditions such as capture effect, unsaturated network, and erroneous channel conditions. In particular, we put emphasis on the effect of PHY capture on the energy efficiency of network. PHY capture is the capability of wireless devices of successfully receiving one frame when two or more than two frames simultaneously arrive [6] at the network interface of receiver. For example, there are two nodes, $S_{1}$ and $S_{2}$ in a network and are $d_{1}$ and $d_{2}$ distance apart from AP as shown in Figure 1 . Both $S_{1}$ and $S_{2}$ transmit to AP. If AP receives packets from both nodes simultaneously, it evaluates the receiving signal strengths of both receiving packets and accepts one of the packet whose signal strength is higher than the other by a certain ratio. This ratio is called capture threshold. Obviously packet from the other node is discarded. Capture effect reduces the number of collisions and increases the number of successful transmissions.

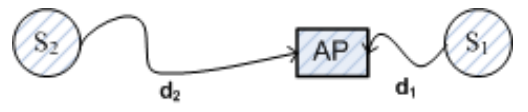

Figure 1. A simple scenario of two nodes communicating with an AP (Access Point) in the presence of physical layer (PHY) capture effect.

In this paper, at first we present an analytical model that takes into account the capture effect; unlike previously proposed analytical models for calculating the energy efficiency of WLAN that ignore taking into account the capture probability [4,7]. We validate the presented analytical model via simulations in various network settings such as different values of capture threshold, erroneous channel and unsaturated traffic load. Secondly, we show that although capture effect improves overall energy efficiency of WLAN by about $20 \%$ but it causes unfairness; a group of nodes located far away from AP is less energy efficient than the group of nodes located closer to the AP.

Finally, to reduce the stated unfairness problem we propose a joint strategy based on adaptive transmission power control (ATXPR) and contention window adjustment (CWADJ). ATXPR additively or multiplicatively increases or decreases transmission power of a node whose transmission is unsuccessful due to capture failure. Capture failure is considered when transmitter does not receive an ACK but instead overhears it. In other words, two or more transmitters send data frames simultaneously but only one of them has successfully captured the channel and therefore only that transmitter receives ACK frame whereas the remaining nodes only overhear the ACK frame. When a node's transmission is unsuccessful because of a capture failure, it (i) increases the transmission power level to the next available transmission power among a set of predefined transmission power 
levels according to additive increase (AI) or multiplicative increase (MI) and (ii) stays in the same backoff stage without increasing its CW according to CWADJ. This increase in power helps the node avoid another capture failure in the next round of transmission. A node after successful transmission decreases its transmission power additively (additive decrease) or multiplicatively (multiplicative decrease) and resets its CW. Our proposed mechanism on one hand increases the probability of successful transmission and on the other hand lets the node conserve energy by not spending more time in contention. Simulation results show that our proposed algorithm is $99 \%$ fair.

This paper is organized as follows: Section 2 presents the literature review. Section 3 explains the analytic model to calculate energy efficiency of WLAN in the presence of capture effect, unsaturated traffic conditions and erroneous channel. Section 4 discusses the results obtained from analytical models and simulation. Section 5 highlights the unfairness caused by the capture effect in terms of energy efficiency and proposes several strategies to overcome the unfairness problem. The proposed strategies are evaluated via simulations and results are compared. Section 6 finally concludes the paper.

\section{Related Works}

Yong He et al. [5] conducted an experimental study using PDA to analyze the power consumption in both PSM and CAM. Authors found that background traffic significantly deteriorates the performance of PSM. From the experiments they observed that PDA network interface changes from PSM to CAM as the traffic rate exceeds a certain limit.

Daewon et al. [7] proposed a power saving strategy based on balancing the energy and delay metrics. Their proposed strategy suggests that AP should restrict the number of nodes in wakeup mode during the beacon interval to achieve better performance. However, their proposed strategy is not useful for CAM as there is no sleep mode in CAM. Anurag Kumar et al. [4] proposed analytic models to measure the energy consumption in a WLAN carrying TCP traffic. Authors also proposed the opportunistic power save mode (OPSM), according to which only one station downloads a file from an AP at any instant whereas all other stations wait for random durations of time to conserve energy [8].

Daji et al. [9] proposed a transmission power control mechanism for the energy efficient operation of PCF. Their proposed mechanism, however, is not suitable for the DCF-based medium access control (MAC) protocol.

Markus et al. [10] conducted experiments on 802.11n testbed and found that PSM is largely ineffective for short inter-packet arrival times. The range of offered loads and traffic patterns used for the experiment show that the opportunity for the interfaces to go into sleep mode decreases.

In [11] authors conducted extensive experiments and observed that PSM suffers significant increase in the energy consumption due to CAM traffic. Authors proposed network assisted power management (NAPman) energy-aware fair scheduler that aims to enhance energy efficiency in PSM clients without causing unfairness to the CAM clients.

All the above literature reviewed either identify the shortcomings of the power saving mechanism of IEEE 802.11 or propose solution for the energy efficient operation of WLAN. However, these solutions are based on MAC and do not take the PHY characteristics into account. Unlike the above studies, some recent research works have explored the use of PHY capture effect to improve the throughput of WLAN. For example, in [6], authors performed experiments and showed that there exists capture effect on wireless interfaces. Moreover in [12-14], authors proposed models for the performance analysis of IEEE 802.11 WLAN in the presence of capture effect.

Most fairness studies concerning energy issues in battery operated networks try to prolong the network lifetime and balance energy consumption. Fair energy consumption is mostly studied in ad hoc wireless local area network taking into account the routing protocols [15] or in sensor networks taking into account cross-layer design between MAC and network layer [16-19]. The same amount of energy consumption amongst nodes is considered as the fairest case in these studies [20]. 
In [21], authors conducted a measurement study on device-to-device communication and found the unfair energy consumption between the two nodes, one of them being master and the other being slave. The authors further proposed the concept of fair role switching to maintain fairness among the nodes.

In $[22,23]$ authors proposed adaptive transmission power control for senders in a wireless body area network (WBAN) based upon the received signal strength (RSSI) value that it receives from the base station. The authors in this work, however, ignored the unfairness in energy consumption among the group of WBAN nodes having higher RSSI compared to the group of WBAN nodes having lower RSSI.

In [24], authors proposed a mechanism called a differentiated-received-power transmit power control (DRP-TPC) according to which a wireless node configures its transmission power based upon path-loss and distance from the access point. DRP-TPC increases the effect of capture at the receiver when simultaneous transmission are received at the receiver. While this mechanism increases overall throughput, it is worth mentioning that it does not address the issue of unfair energy consumption among the groups of nodes present in the network.

In $[25,26]$ authors proposed transmission power control mechanisms to increase energy efficiency in multi-hop wireless networks. Their propsed mechanism is based on link scheduling and routing. This work, however, does not address the issue of unfair energy consumption among the nodes that may have been caused by the use of transmission power control.

Finally, in [27] authors proposed a three dimensional Markov chain that takes into account the capture effect as well as various transmission power levels with which a node may transmit. This work, too, like many previous works focuses on the overall saturation throughput. It does not address the issue of unfair energy consumption among the various groups of nodes in the network.

In contrast to the above studies, our work presents a comprehensive energy consumption analysis of IEEE 802.11-based wireless networks in the presence of PHY capture effect. In this research work:

- We show that although capture effect improves overall energy efficiency of the wireless network, it causes unfairness; a group of nodes located farther away from the AP consumes more energy as compared to the group of nodes located closer to the AP.

- For the fair energy consumption among the groups of nodes, we propose a mechanism that is based on transmission power control and contention window adjustment technique.

- We show that the proposed solution lets the nodes spend their energy resources fairly without sacrificing the overall energy efficiency of the network.

- Unlike the notion of fairness used in the literature being 'equal energy consumption', our work uses Jain's Fairness Index [28].

\section{Analytical Model}

This section explains an analytic model which is based on the model presented in [29]. However, unlike the model presented in [29], our proposed model takes into account capture effect in addition to unsaturated traffic condition and erroneous channel as shown in Figure 2. Figure 2 shows that backoff stage increases due to two reasons (i) when two or more frames arrives at the receiver while none of them has significantly higher receiving power than that of all the other remaining frames to successfully capture the channel (ii) when a transmission is significantly impaired by the channel errors. In contrast, a node after successful transmission reaches to state ' $\mathrm{E}$ ' if its transmission queue is empty or randomly selects a backoff counter from the initial contention window $(C W)$ if there is at least one data frame waiting for transmission in its transmission queue.

Table 1 shows the list of notations used in the derivation of the main equations. 
Table 1. Notations and their descriptions.

\begin{tabular}{cc}
\hline Notation & Description \\
\hline$P_{t x}$ & Power consumption during transmission. \\
$P_{r x}$ & Power consumption during reception. \\
$P_{\text {idle }}$ & Power consumption in idle mode. \\
$T_{S}$ & Time spent in successful transmission. \\
$T_{C}$ & Time spent due to frame collision. \\
$T_{E}$ & Time spent in transmission failure due to error. \\
$\sigma$ & Idle slot time. \\
$E_{S}$ & Energy consumption during successful transmission. \\
$E_{c}$ & Energy consumption due to collision. \\
$E_{E}$ & Energy consumption in transmission failure due to error. \\
$E_{i d l e}$ & Energy consumption during idle time slot. \\
$P$ & Probability of transmission failure. \\
$P_{t r}$ & Probability of channel being busy. \\
$W$ & Initial size of the contention window. \\
$\lambda$ & Frame arrival rate with Poisson distribution. \\
$P_{\text {Cap }}$ & Frame capture probability. \\
$P_{c}$ & Probability of frame collision. \\
$P_{e}$ & Probability of transmission failure due to channel error. \\
$P_{S}$ & Probability of successful transmission. \\
$\tau$ & Probabilit of attempt by a node. \\
$\eta$ & Energy efficiency of network in Mb/j. \\
$\eta_{1}$ & Energy efficiency of nodes in Zone 1. \\
$\eta_{2}$ & Energy efficiency of nodes in Zone 1. \\
$N$ & Total number of nodes in network. \\
$N_{1}$ & Total number of nodes in Zone 1. \\
$N_{2}$ & Total number of nodes in Zone 2. \\
$\mu_{f a i r}$ & Fairness index for energy consumption among different zones. \\
&
\end{tabular}

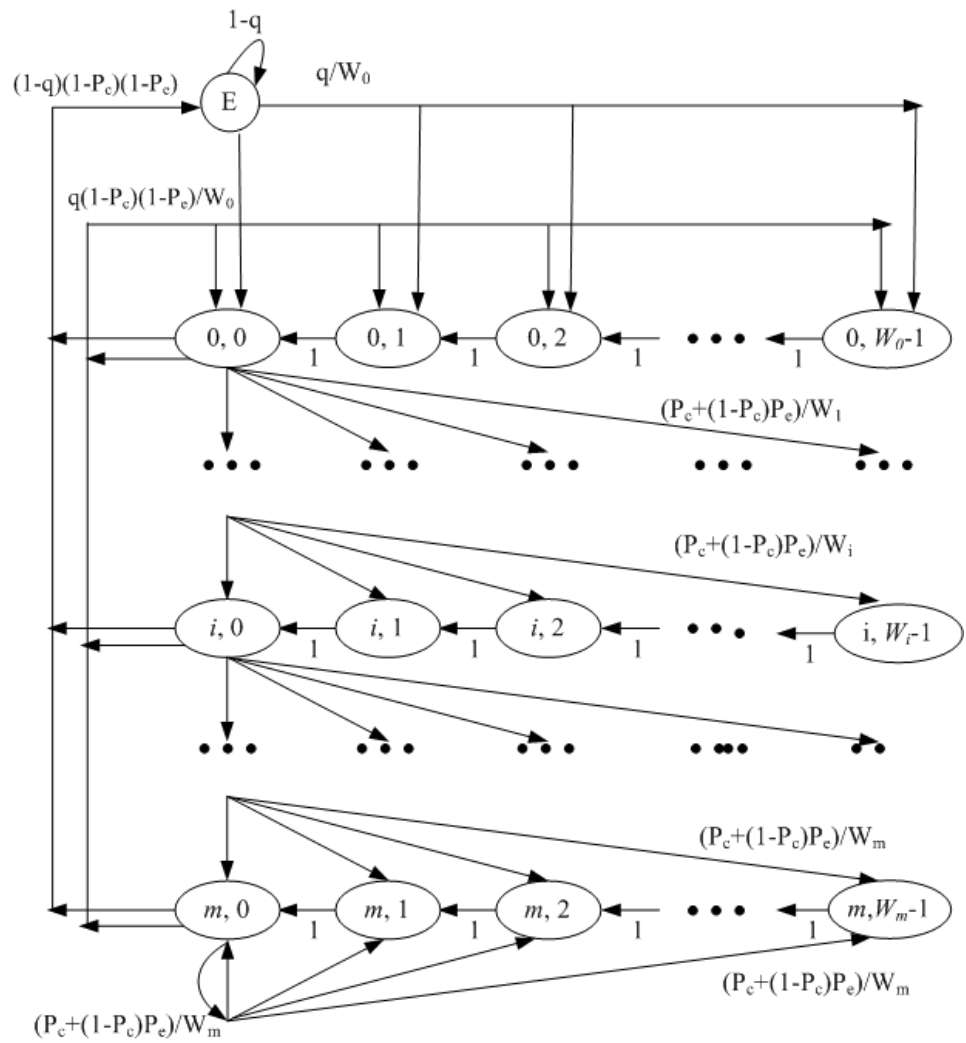

Figure 2. A two dimensional Markov chain model that takes into account capture probability. 
There are two key probabilities.

(1) A node attempts transmission in a generic time slot with probability $\tau$ and is constant across all time slots.

(2) Probability of collision, represented as $P_{c}$ is constant and independent of the number of collisions already suffered and includes probability that the node does not capture the channel.

Now $\tau$ when the channel is unsaturated is given as:

$$
\tau=\frac{2(1-2 P) q}{q\left((1-2 P)(W+1)+W P\left(1-(2 P)^{m}\right)\right)+2(1-q)(1-P)(1-2 P)}
$$

where $W$ and $m$ represent the initial size of $C W$ and the maximum backoff stage. $q$ is the probability that there is at least one frame in the transmission queue of the tagged node, whereas $P$ represents the probability of transmission failure. Parameters $q$ and $P$ are given as:

$$
\begin{gathered}
q=1-e^{-\lambda T} \\
P=P_{c}+P_{e}-P_{c} P_{e},
\end{gathered}
$$

where $\lambda$ in Equation (2) is the frame arrival rate with Poisson distribution and $T$ is the mean slot time. In Equation (3) $P_{e}$ and $P_{c}$ denote the probability that a transmission is unsuccessful due to erroneous channel and collision respectively, and can be expressed as:

$$
\begin{gathered}
P_{e}=1-(1-B E R)^{D} \\
P_{c}=1-(1-\tau)^{(N-1)}-P_{c a p} .
\end{gathered}
$$

BER and D in Equation (4) are bit error rate and size of payload in bits, respectively, whereas $P_{c a p}$ is the frame capture probability and is obtained by summing the contributions from all combinations of the rest of the network nodes transmitting or not. $P_{c a p}$ can be obtained as follows:

$$
P_{c a p}=\sum_{i=1}^{N-1}\left(\begin{array}{c}
N \\
i+1
\end{array}\right) \tau^{i+1}(1-\tau)^{N-1-i} P_{c p}\left(S I R>Z_{0} g\left(S_{f}\right) \mid i\right)
$$

$P_{c p}\left(\operatorname{SIR}>Z_{0} g\left(S_{f}\right) \mid \mathrm{i}\right)=\frac{1}{\left[1+Z_{0} g\left(S_{f}\right)\right]^{i}}$ in Equation (6) is the conditional capture probability where SIR is the signal to interference ratio of the strongest signal represented as $P_{r}$ to the $i$ interfering signals such that SIR $=\frac{P_{r}}{\sum_{n=1}^{i} P_{r(n)}}$. To find $P_{c p}$, we used the capture model that is derived under the hypothesis of power-controlled stations in infrastructure mode and Rayleigh fading [30,31]. The function $g\left(s_{f}\right)$ is the inverse processing gain where $s_{f}=11$ for using the direct sequence spread spectrum (DSSS) and $Z_{0}$ is the capture threshold. For detailed derivation of $P_{c p}$ readers are refered to [30]. The mean slot time, $T$, is expressed as:

$$
T=P_{t r} P_{S}\left(1-P_{e}\right) T_{S}+P_{t r}\left(1-P_{S}\right) T_{C}+P_{t r} P_{S} P_{e} T_{E}+\left(1-P_{t r}\right) \sigma,
$$

where $P_{t r}=1-(1-\tau)^{N}$ is the probability that there is at least one transmission in the network in a randomly chosen time slot. $P_{S}=\frac{N \tau(1-\tau)^{(N-1)}+P_{\text {cap }}}{P_{\text {tr }}}$ is the successful conditional probability that a transmission is successful and corresponds to the case when exactly one frame is transmitted, or when more than one frames are transmitted simultaneously that result in successful frame capture. $T_{S}, T_{C}$ and $T_{E}$ are the time durations for the successful frame transmission, transmission failure due to collision and channel interference, respectively, and are given as:

$$
T_{S}=D I F S+T_{D}+S I F S+T_{A}
$$




$$
\begin{aligned}
& T_{C}=T_{D}+\text { EIFS } \\
& T_{E}=T_{D}+\text { EIFS }
\end{aligned}
$$

where $T_{D}$ and $T_{A}$ are data frame transmission time including the PHY and MAC headers, and ACK transmission time, whereas EIFS $=$ SIFS $+T_{A}+$ DIFS.

Now that we have derived some basic expressions, energy efficiency, denoted as $\eta$, of the considered WLAN in CAM can be expressed as:

$$
\eta=\frac{E[L]}{E}
$$

where $E[L]=P_{t r} P_{S}\left(1-P_{e}\right) \mathrm{L}$ is the amount of payload successfully transmitted in the network, and $\mathrm{E}$ is the total energy consumed by the network and is the sum of the energies spent in the four instances, namely successful transmission, collision, failed transmission due to channel interference and the time when nodes were idle, and is given as:

$$
E=P_{t r} P_{S}\left(1-P_{e}\right) E_{S}+P_{t r}\left(1-P_{S}\right) E_{C}+P_{t r} P_{S} P_{e} E_{E}+\left(1-P_{t r}\right) E_{I} .
$$

Below are the derived expressions for $E_{S}, E_{C}, E_{E}$, and $E_{I}$

$$
\begin{gathered}
E_{S}=T_{D}\left[N_{c a p} P_{t x}+\left(N-N_{c a p}\right) P_{r x}\right]+N T_{A} P_{r x}+N P_{\text {idle }}[S I F S+D I F S] \\
E_{C}=T_{D}\left[N_{c o l} P_{t x}+\left(N-N_{c o l}\right) P_{r x}\right]+N T_{A} P_{r x}+N P_{\text {idle }}[E I F S] \\
E_{E}=T_{D}\left[P_{t x}+(N-1) P_{r x}\right]+N P_{\text {idle }}[\text { EIFS }] \\
E_{\text {idle }}=N P_{\text {idle }} \sigma,
\end{gathered}
$$

where $P_{t x}, P_{r x}, P_{\text {idle }}$ are the transmission, reception and idle mode power consumption (in Watts), respectively, and $N_{c a p}$ is the average number of simultaneously transmitted frames resulting in atleast one successful transmission due to capture with probability $P_{c a p}$ and $N_{c o l}$ is the average number of frames transmitted simultaneously resulting in collision. $N_{c a p}$ and $N_{c o l}$ can be calculated as:

$$
\begin{gathered}
N_{\text {cap }}=\sum_{i=1}^{N-1} i\left(\begin{array}{c}
N \\
i+1
\end{array}\right) \tau^{i+1}(1-\tau)^{N-1-i} P_{c p}\left(S I R>Z_{0} g\left(S_{f}\right) \mid i\right) \\
N_{c o l}=\frac{\sum_{n=2}^{N} n\left(\begin{array}{c}
N \\
n
\end{array}\right) \tau^{n}(1-\tau)^{N-n}}{P_{t r}\left(1-P_{S}\right)} .
\end{gathered}
$$

\section{Results and Analysis}

In this Section, we perform experiments using ns-2 simulator [32]. Table 2 shows the values used for various parameters during mathematical calculations and simulation of the IEEE 802.11g type of WLAN. Network consists of 30 nodes and an AP. All 30 nodes were randomly scattered around AP in a circle with a radius of $100 \mathrm{~m}$ and they transmited data frames of size $L=1000$ bytes.

Figure 3 shows the energy efficiency of WLAN when the number of nodes in network is fixed as 30. The traffic load varied from 100 to 1500 frames/s and the channel was error free, i.e., $P_{e}=0$. We took three different values for the capture threshold, i.e., $Z_{0}=\{\infty, 10 \mathrm{~dB}, 6 \mathrm{~dB}\}$. When $Z_{0}=\infty$, capture did not occur. In such situation, the network gave the lowest energy efficiency as compared to the other two values of $Z_{0}$. In contrast, the network was more energy efficient when $Z_{0}=6 \mathrm{~dB}$, which shows that capture happened more frequently than when $Z_{0}=10 \mathrm{~dB}$. Figure 3 also shows that, initially, the energy efficiency values were close to each other when the network was lightly loaded, that is, $\lambda=100 \mathrm{frames} / \mathrm{s}$, because the probability of two or more than two frames arriving simultaneously 
in same time slot was very low. This indicates that capture was not very effective in unsaturated conditions. As the network approached mild saturated conditions such as $\lambda=500$ frames/s or above the effect of capture was more obvious. While in mild saturated condition, that is, $\lambda=\{500 \sim 1500\}$ frames/s, energy efficiency remained almost the same in the presence of capture, it started decreasing more steeply in the absence of capture, that is, when $Z_{0}=\infty$. The simulation results (abbreviated as Sim in the legend of Figure 3) matched the results obtained from the analytical model (abbreviated as Mod in the legend of Figure 3) and thereby validated the accuracy of our analytical model.

Table 2. Parameters for experiments.

\begin{tabular}{cc}
\hline Parameter & Value \\
\hline Data rate & $54 \mathrm{Mbps}$ \\
Control rate & $6 \mathrm{Mbps}$ \\
PHY header & $20 \mu \mathrm{s}$ \\
MAC header & $272 \mathrm{bits}$ \\
ACK frame & $112 \mathrm{bits}$ \\
Slot time $\sigma$ & $9 \mu \mathrm{s}$ \\
CW $W_{\text {min }}$ & 15 \\
Maximum backoff stage $(\mathrm{m})$ & 5 \\
Transmit power $\left(P_{t x}\right)$ & $2000 \mathrm{~mW}$ \\
Receive power $\left(P_{r x}\right)$ & $1000 \mathrm{~mW}$ \\
Idle power $\left(P_{i d l e}\right)$ & $1000 \mathrm{~mW}$ \\
\hline
\end{tabular}

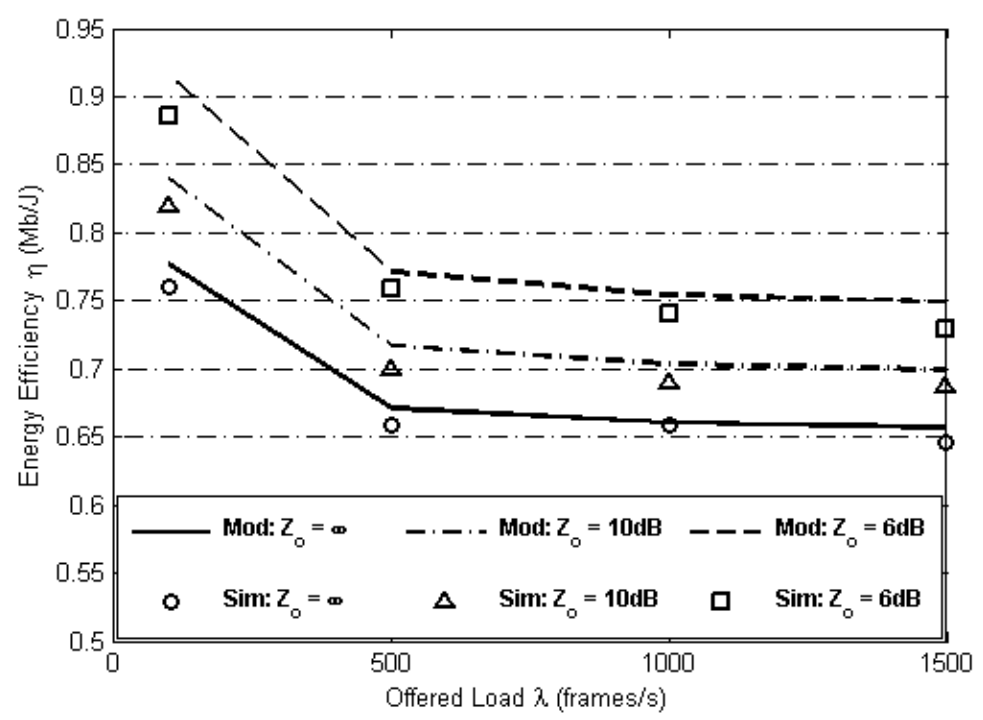

Figure 3. Energy efficiency in presence of capture against varying offered load.

Figure 4 shows energy efficiency of WLAN when the number of nodes in network is 30, $Z_{0}=\{\infty, 10 \mathrm{~dB}, 6 \mathrm{~dB}\}, \lambda=1500$ frames $/ \mathrm{s}$ and $P_{e}=\{0,0.1,0.2,0.3\}$. As previously stated, $Z_{0}=\infty$ corresponds to the absence of capture and therefore gives the lowest energy efficiency. Energy efficiency decreased more when channel was not ideal, i.e., when $P_{e}=0.3$. Furthermore, in the presence of capture, for example, for the same values of $Z_{0}=10 \mathrm{~dB}, 6 \mathrm{~dB}$, the network gave lower energy efficiency when $P_{e}=0.3$ as compared to the case when $P_{e}=0.1$. However, energy efficiency in the presence of capture was still higher than the case when there was no capture effect in the network. This experiment indicates that taking into account channel condition is important for the study of capture effect. 


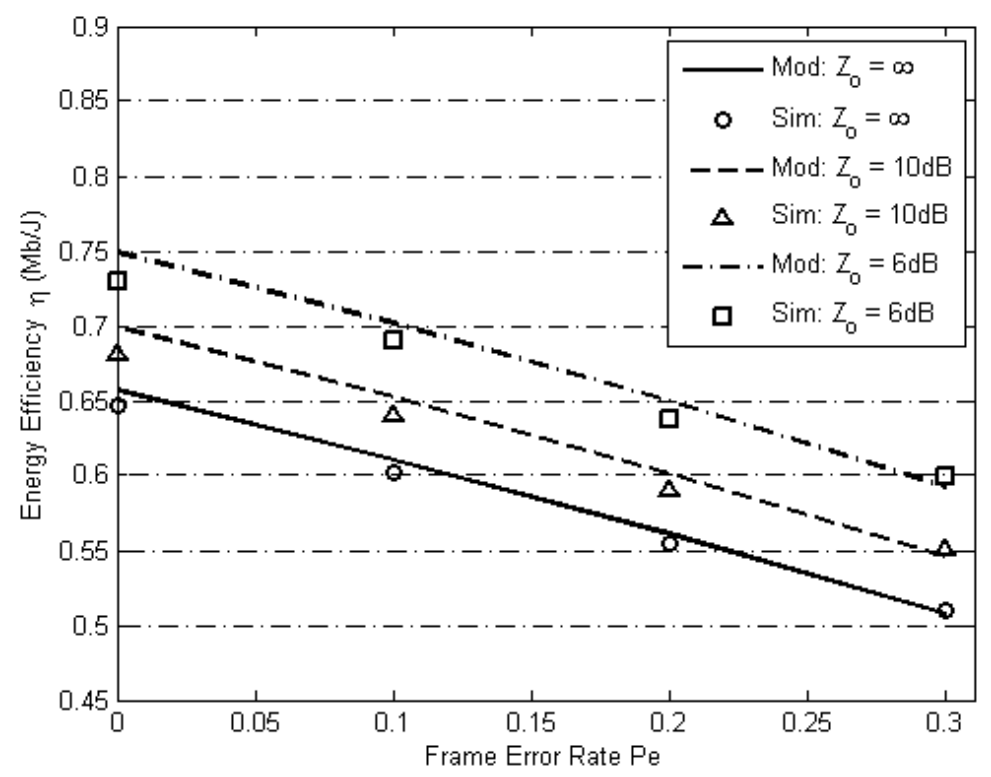

Figure 4. Energy efficiency against varying frame error rate in the presence and absence of capture.

Figure 5 shows the effect of three different values of capture threshold, i.e., $Z_{0}=\{20 \mathrm{~dB}, 10 \mathrm{~dB}, 6 \mathrm{~dB}\}$, frame error rate, $P_{e}=\{0,0.1,0.2\}$ when $N=30$ and when the network was fully saturated, i.e., $\lambda=\infty$. At first, it can be seen in the figure that energy efficiency is always high when channel is ideal, i.e., $P_{e}=0$ irrespective of the underlying value of capture threshold. However, in the presence of erroneous channel i.e., when $P_{e}=0.3$, network gives better throughput for $Z_{0}=6 \mathrm{~dB}$ as compared to the case when $Z_{0}=20 \mathrm{~dB}$ again indicating the importance of capture effect in saturated network and in the presence of erroneous channel conditions.

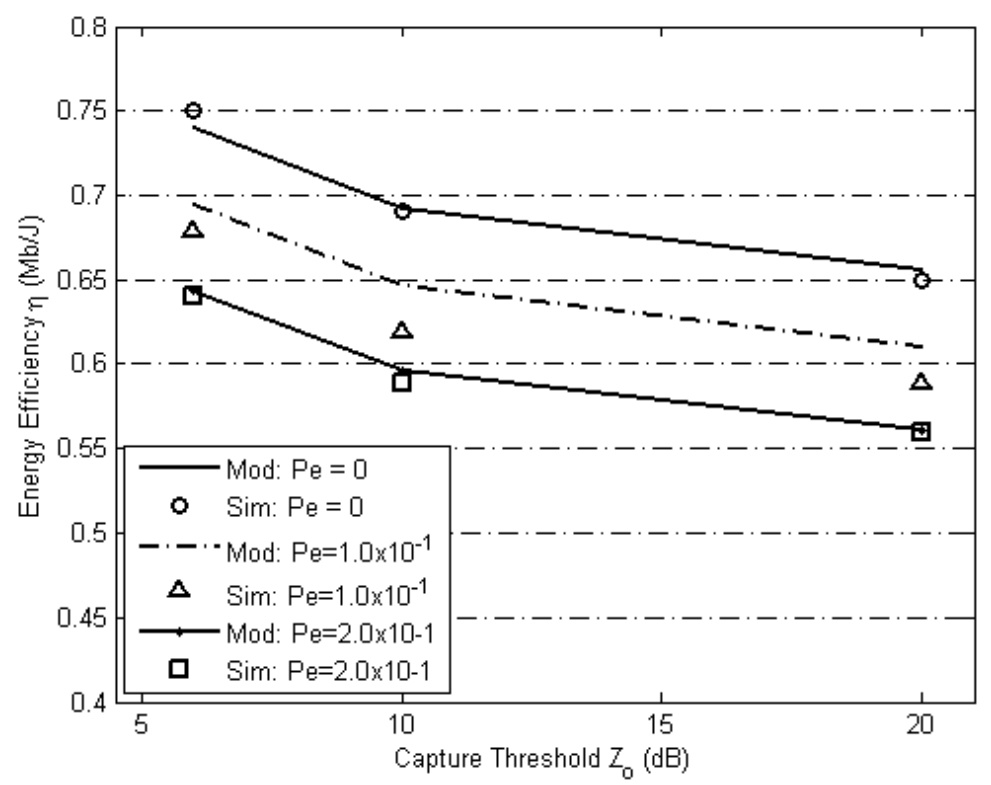

Figure 5. Energy efficiency in the presence and absence of channel error against varying value of $Z_{0}$.

Finally, Figure 6 shows the energy efficiency of network for varying number of nodes in network when the queues of all nodes are saturated in the presence and absence of PHY capture. The effect of capture is obvious for all values of $N$. The gap between the energy efficiencies when there were 10 nodes in the network was maximum, i.e., in presence and absence of capture. Thus showing improvement in energy efficiency due to capture effect. For a network of size 30 nodes, this improvement in energy 
efficiency was about $20 \%$ when network is fully saturated. Hence taking into account all the previous analysis it is obvious that capture is an effective phenomenon to enhance the energy efficiency of IEEE 802.11 based WLANs. In next section we discuss some scenarios in which capture effect improves overall energy efficiency of WLAN but at the cost of unfairness in terms of energy efficiency. We also propose solutions to reduce the unfairness problem.

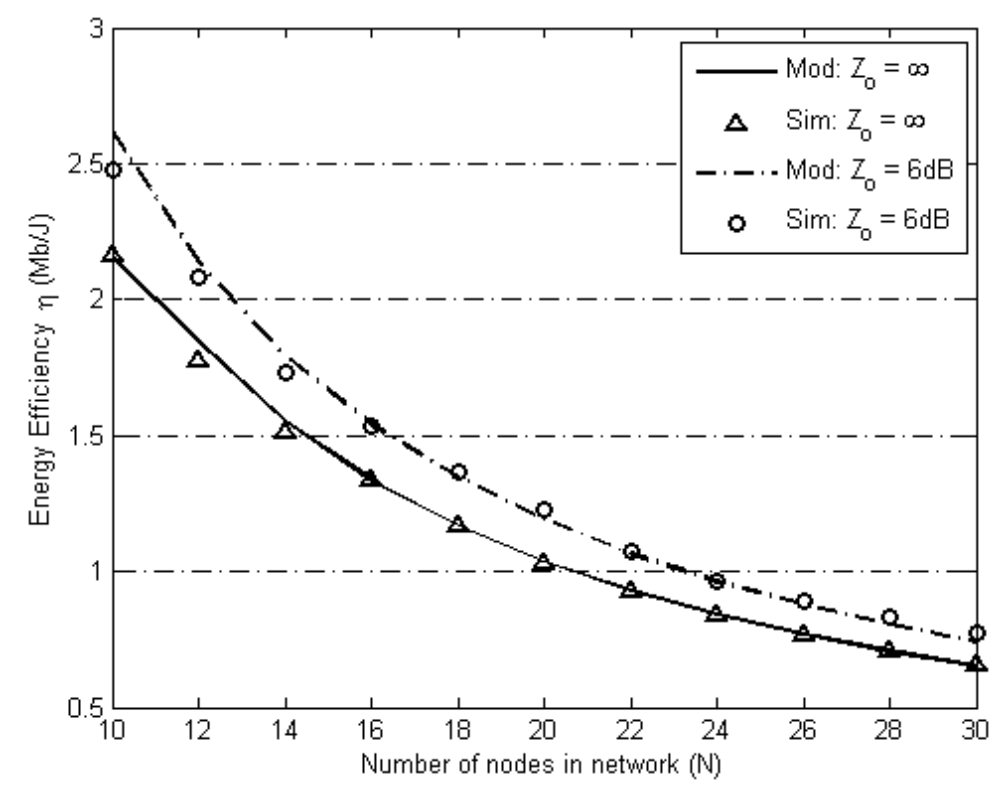

Figure 6. Energy efficiency in the presence and absence of capture effect with varying number of nodes in network.

\section{PHY Capture Induced Unfairness in WLAN and Proposed Solutions Based on Joint Strategy}

In previous sections we showed that capture effect improves the overall network energy efficiency. In this section, we show that although capture effect increases the overall energy efficiency of a WLAN, it it results in unfairness, i.e., the group of nodes located closer to the AP is twofold more energy efficient than the group of nodes located far away from AP.

To understand the unfairness caused by the capture effect, we expanded the scenario given in Figure 1 by increasing the number of nodes in the network as shown in Figure 7 and broadly dividing the network geographically into two zones. We call the area closer to the AP Zone 1 and the area at farther distance from AP is called Zone 2. When two nodes, one located in Zone 1 and other located in Zone 2, transmit packets simultaneously, the former being closer to AP successfully captures the channel, while the latter's packet is dropped causing node from Zone 2 to increase its $\mathrm{CW}$. Hence, long run nodes located in Zone 1 transmit more data to AP consuming almost the same or less amounts of energy than the nodes located in Zone 2. In addition, Zone 2 nodes spend more energy in contention and overhearing transmissions between Zone 1 nodes and AP. Zone 1 nodes successfully transmit only if one of them wins contention and none of the Zone 2 nodes transmit in the same time slot. We demonstrate the effect of capture on unfairness in energy efficiency by performing some simulations.

the simulation scenario consists of WLAN as shown in Figure 7 having AP in the centre of the network. Let the total number of nodes in network be $N_{\text {total }}=20$ and number of nodes in Zone 1 and Zone 2 are $N_{1}=10$ and $N_{2}=10$, respectively. Groups of nodes in Zone 1 and Zone 2 surround $\mathrm{AP}$ at distances of $40 \sim 50 \mathrm{~m}$ and 70 140 $\mathrm{m}$ from AP, respectively. Traffic in both zones is saturated, transmission failure due to erroneous channel is negligible and $L=2000$ bytes.

To measure fairness, we used Jain's fairness index proposed in [28]. Jain's fairness index has been widely used as a representative fairness index and the resulting value is bounded between 0 and 1 
and denoted as $\mu_{\text {fair }}$. Let $\eta_{1}$ and $\eta_{2}$ are the energy efficiencies of group of nodes located in Zone 1 and Zone 2, respectively, then $\mu_{\text {fair }}$ according to Jain's fairness index among the two groups of nodes is

$$
\mu_{\text {fair }}=\frac{\left(\eta_{1}+\eta_{2}\right)^{2}}{2\left(\eta_{1}^{2}+\eta_{2}^{2}\right)}
$$

Figure 7. Wireless local area networks (WLANs) division in various zones depending on their respective distances from AP.

Simulations were performed with and without the capture effect as shown in Figure 8. At first, Figure 8 shows that in the absence of capture effect, i.e., $Z_{0}=\infty$, energy efficiency of both zones is almost equal, i.e., $\mu_{\text {fair }}=1$ but this fairness comes at the cost of lower aggregate energy efficiency, i.e., $\eta=1.30$. In the presence of the capture effect, when $Z_{0}=6 \mathrm{~dB}$, although the aggregate energy efficiency was improved, i.e., $\eta=1.51$, but this caused unfairness, i.e., energy efficiency of the group of nodes in zone 2 was only $\eta_{1}=0.7 \mathrm{Mb} / \mathrm{J}$ as compared to energy efficiency of the group of nodes in zone 1 which was $\eta_{1}=2.2 \mathrm{Mb} / \mathrm{J}$.

In the presence of the capture effect, according to (19) $\mu_{\text {fair }}=0.81$ which shows significant unfairness. We call the results obtained from the simple capture effect as baseline and will compare them with the results obtained using various joint strategies in the following sections.

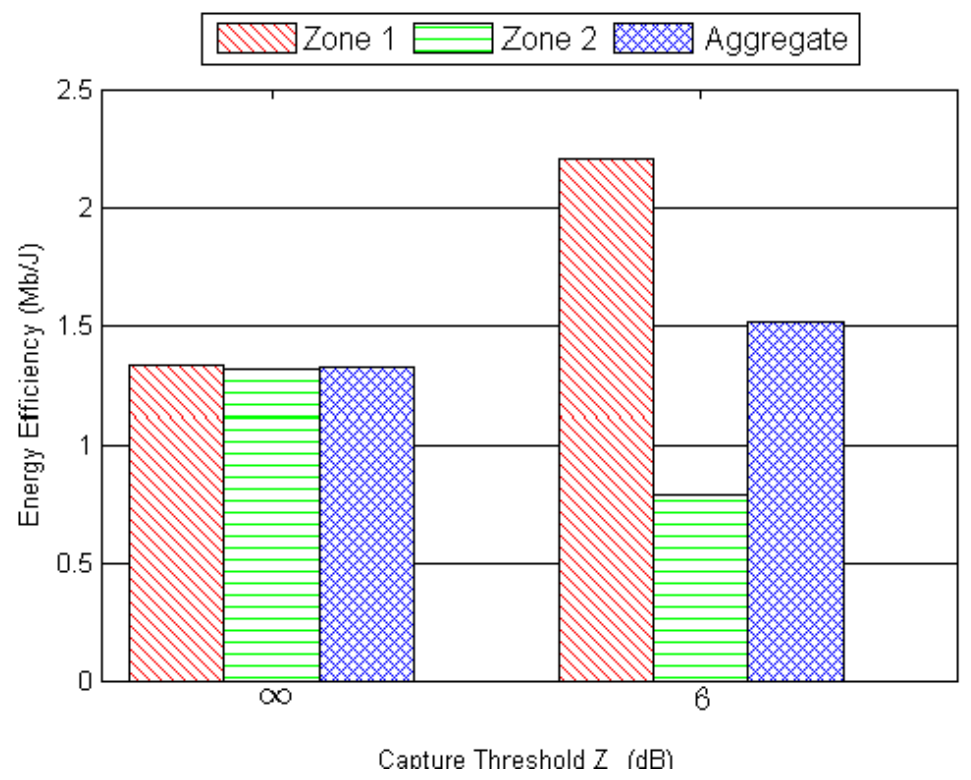

Figure 8. Energy efficiency comparison for different values of capture threshold. 


\subsection{Contention Window Adjustment Technique (CWADJ)}

According to this strategy, node(s) suffering an unsuccessful transmission due to capture failure refrain from increasing the size of their $\mathrm{CW}$, whereas in case of transmission failure due to collision, the tagged node increases the size of its $C W$ according to BEB. The idea behind choosing the same $\mathrm{CW}$ when a transmission failed due to capture failure is to compensate the node for spending energy in contention and overhearing transmissions in the preceding round.

Note that transmission failure due to capture is different than collision; the former happens when transmission of the tagged node is failed due to the successful capture of another transmission whereas the latter happens when all the simultaneously arriving packets in the same slot are destroyed. The mechanism behind recognition of whether a transmission is failed due to capture failure is the overhearing of the ACK frame transmitted from the AP to the sender node whose packet has successfully captured the channel. In contrast, during collision no ACK frame is received or overheard during the ACK time out.

Hence, if a node after it transmits a data frame overhears an ACK which is destined for another node in the network declares a capture failure and randomly chooses a backoff counter without increasing its retransmission counter. This strategy gives the node suffered from capture failure the opportunity to conserve its energy by not staying for longer duration in contention. However, a node declares collision if it neither overhears nor receives an ACK frame after it transmits a data frame and would therefore increases its backoff stage for the next transmission attempt.

\subsection{Adaptive Transmission Power Control (ATXPR)}

We defined a set of 10 transmission power levels as shown in Table 3. According to ATXPR, initially every node transmited with the default transmission power denoted as DefaultTxPwrLevel. When a node suffered a transmission failure due to capture failure it invoked the adaptive transmission power control mechanism. In detail, when a transmission from a node in Zone 2 was unsuccessful because of capture failure at the AP, according to ATXPR this node stepped up its transmission power level for the next transmission attempt. This transmission power control strategy is only activated at the onset of transmission failure due to capture failure. This increase in transmission power level can be additive i.e., additive increase (AI) or multiplicative, i.e., multiplicative increase (MI). A node increased the level of transmission power as long as transmission suffers from capture failure or until the highest level of transmission power is reached at which point it will maintain the same highest power level as shown in Table 3.

Table 3. Transmission power levels for adaptive transmission power control.

\begin{tabular}{cc}
\hline Retransmission Counter & Power Level (dBm) \\
\hline 0 & 10.54 \\
1 & 12.62 \\
2 & 14.91 \\
3 & 18.08 \\
4 & 20.23 \\
5 & 22.5 \\
6 & 24.62 \\
7 & 26.91 \\
8 & 27.08 \\
9 & 28.23 \\
10 & 30.5 \\
\hline
\end{tabular}

A node that has increased its transmission level decreases either additively, i.e., additive decrease (AD) or multiplicatively, i.e., multiplicative decrease (MD) only when its transmission was successful. However, after collision, a node neither increased nor decreased its transmission power level. 
In summary, we had four different configurations of transmission power adjustment, i.e., AIAD, AIMD, MIAD and MIMD.

The above four mentioned transmission power control techniques of ATXPR in combination with the CWADJ jointly result in four distinct algorithms; additive increase and additive decrease (AIAD+), additive increase and multiplicative decrease (AIMD+), multiplicative increase and additive decrease (MIAD+) and finally, multiplicative increase and multiplicative decrease (MIMD+). The ' + ' symbol appended at the end of each of the above terms shows the addition of CWADJ to the four adaptive transmission power control algorithms. Note that all the four adaptive transmission power control algorithms operate in the presence of capture effect.

These strategies helped the node suffering from capture failure to conserve its energy by staying in idle mode for a comparatively lesser duration during the contention and it also increased its chance of successfully capturing the channel in case another node transmits simultaneously in the next round of transmission.

\subsubsection{Performance Evaluation of Proposed Solutions}

Figure 9 compares the five proposed solutions, i.e., CWADJ, AIAD+, AIMD+, MIAD+ and MIMD+ with the baseline.

As shown in Figure 9 CWADJ improved fairness in energy efficiency, though limited, without sacrificing the overall aggregate energy efficiency of the network. Jain's fairness index for CWADJ according to Equation (13) was 0.91 , which was almost $12 \%$ improvement in fairness compared to the baseline. The limited improvement in fairness is because CWADJ mildly increases the number of transmission opportunities of Zone 2 nodes, however, Zone 2 nodes still suffered more transmission failures due to capture failure when their transmission overlap with the transmissions from Zone 1 nodes.

Among the four adaptive algorithms, AIMD+ gave the best performance in terms of fairness whereas MIMD+ gives best performance in terms of aggregate energy at the cost of fairness. Overall all the four adaptive transmission power control algorithms performed better than the baseline and CWADJ. AIMD+ improved fairness by $22 \%$ and $8 \%$ compared to the baseline and CWADJ, respectively.

The reason behind AIMD+ is that the slow increase in the transmission power gives more opportunity of capture for the simultaneously arriving packets. In contrast, the quick increase in transmission power in MIMD+ causes more collisions than capture for the transmissions from the nodes located far away from the AP. Table 4 compares the fairness and efficiency for all the six methods.

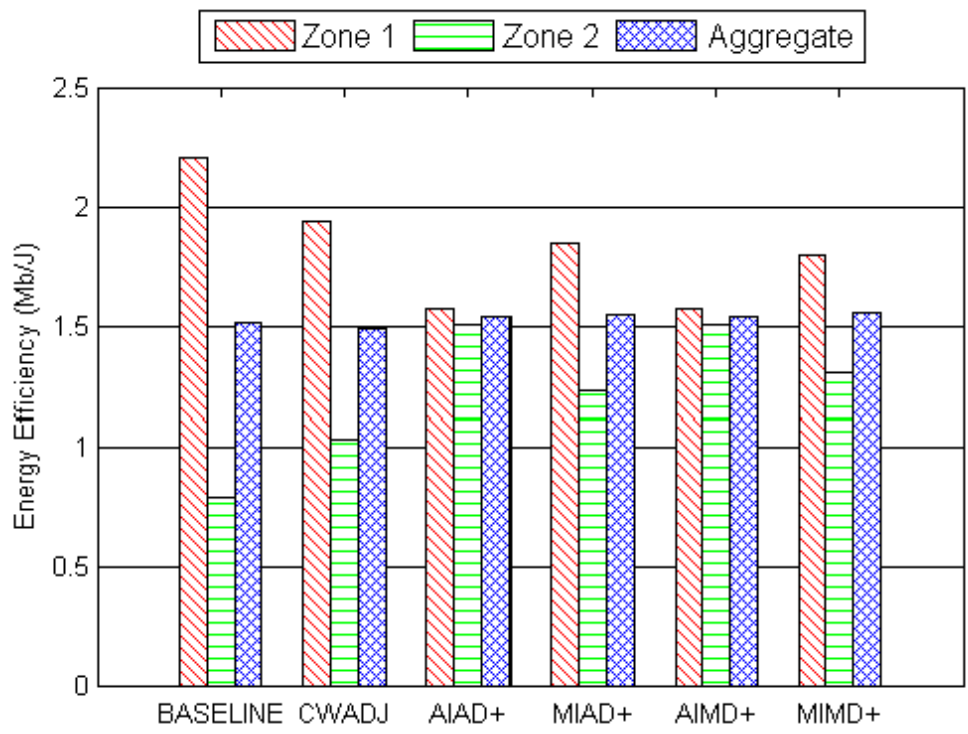

Figure 9. Comparison of three proposed strategies with the baseline. 
Table 4. Result comparisons.

\begin{tabular}{ccc}
\hline Strategy & $\boldsymbol{\mu}_{\text {fair }}$ & $\boldsymbol{\eta}$ \\
\hline Baseline & 0.81 & 1.51 \\
CWADJ & 0.91 & 1.51 \\
AIAD+ & 0.98 & 1.53 \\
MIAD+ & 0.96 & 1.54 \\
AIMD+ & 0.99 & 1.54 \\
MIMD+ & 0.97 & 1.55 \\
\hline
\end{tabular}

To validate our argument of AIMD+ being superior to the rest of the three presented algorithms we determined the collision probability of nodes in each of the two zones and compare them in Figure 10. It can be seen from Figure 10 that AIMD+ significantly reduced the collision probability of Zone 2 nodes followed by AIAD+, MIAD+ and MIMD+. The feature of additive increase in transmission power in both AIMD+ and AIAD+ effectively reduced the collision probability in Zone 2 nodes and thereby increased fair energy consumption and efficiency whereas as the multiplicative increase in MIAD+ and MIMD+ increased the collision probability of Zone 2 nodes. From Figures 9 and 10, it can be concluded that AIMD+ performs better than the baseline and the remaining three transmission and $\mathrm{CW}$ control algorithms, i.e., MIAD+, AIMD+, and MIMD+. We also performed simulations when there were for more than two zones in the network and found similar results as above. Due to space limitation we do not illustrate those results here. Instead we performed some extended simulations and illustrate the obtained results in the next subsection.

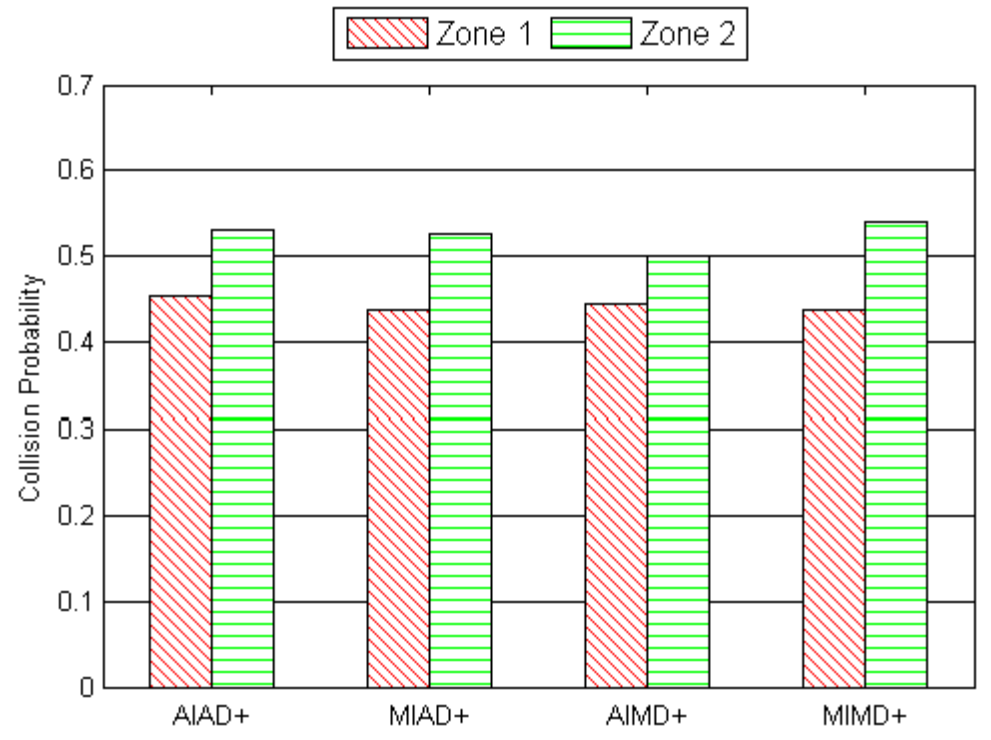

Figure 10. Collision probability comparison between four transmission control algorithms.

\subsubsection{Extensive Simulations}

In the remaining paper we choose AIMD+ and compared its performance with the baseline in various settings. We compared the energy efficiency obtained by nodes in Zone 1, Zone 2, and aggregate network energy efficiency for the baseline and AIMD+, shown in Figure 11. In this experiment, the total number of nodes in the network remained constant, i.e., $N_{\text {total }}=20$ and the number of nodes in Zone 1 and Zone 2, denoted as $N_{1}$ and $N_{2}$, respectively, varied from 2 18. At first, this comparison shows that the aggregate network efficiency, for both baseline and AIMD+, overlap each other and that our proposed solution does not compromise on the aggregate energy efficiency. Secondly, energy efficiency of nodes in Zone 2 when using AIMD+ always remains higher than the baseline method for any value of $N_{2}$. Thirdly, when using the AIMD+ mechanism energy efficiencies for both Zone 1 nodes 
and Zone 2 nodes approach convergence when $N_{1}=N_{2}=10$, indicating AIMD+ is substantially fairer than the baseline.

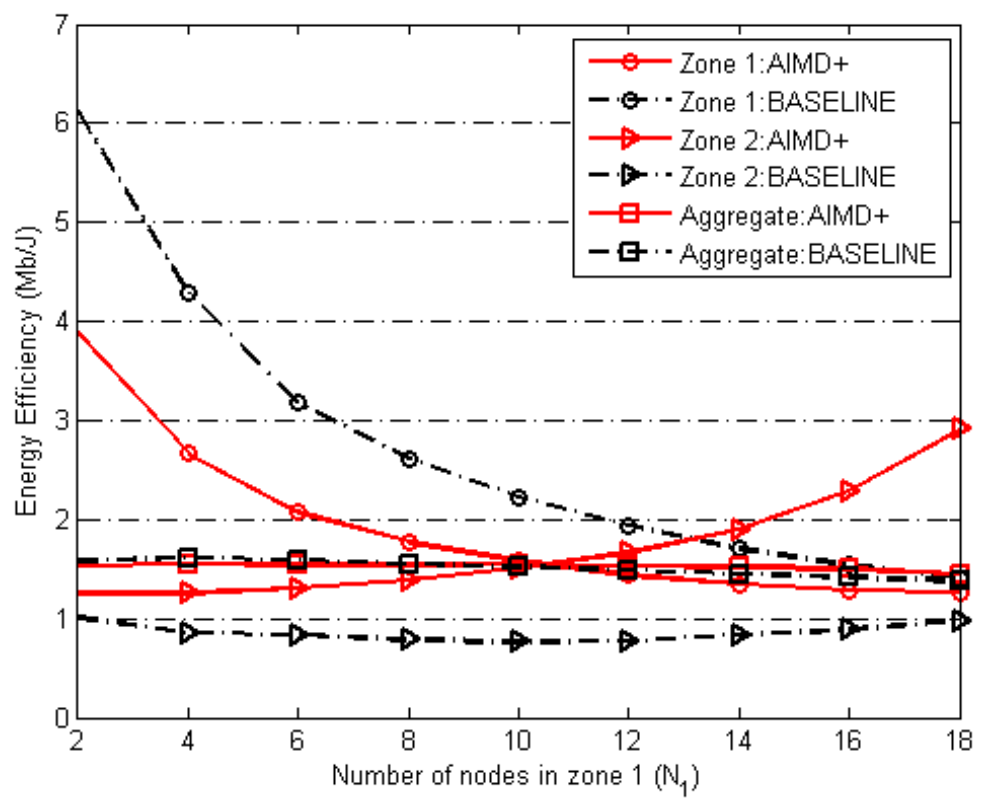

Figure 11. Comparison of additive increase, multiplicative decrease (AIMD+) with baseline.

Figure 12 compares energy efficiencies obtained using AIMD+ and baseline with varying inter-zone distances. Similar to Figure $11, N_{\text {total }}=20$ whereas $N_{1}$ and $N_{2}$ are fixed to 10 . At first, we see the overlap between the aggregate energy efficiency obtained using AIMD+ and baseline. It means our proposed solution is effective in maintaining the overall aggregate energy efficiency. Secondly, we see that when the inter-zone distance was initially $20 \mathrm{~m}$, there was no difference between the energy efficiencies obtained via baseline and AIMD+ for both zones. The reason is that at $20 \mathrm{~m}$ difference there are very few successful captures. Most simultaneous transmissions turn into collisions, hence, AIMD+ is rarely invoked. Moreover, as the inter-zone distance starts increasing capture starts happening frequently that causes unfairness between the energy efficiency of Zone 1 and Zone 2 using the baseline. At $40 \mathrm{~m}$ inter-zone distance this unfairness reaches its peak. In contrast to baseline our proposed solution improves efficiency of Zone 2 nodes in addition to keeping unfairness to minimum unlike baseline. Even though energy efficiency of the group of nodes in Zone 1 decreases using AIMD+, overall energy efficiency remains same and fairness improves to the maximum level for all values of inter-zone distance.

We also compare the throughput performance obtained by baseline and AIMD+ in Figure 13. Similar to Figure 11 the total number of nodes in network is $N_{\text {total }}=20$ and $N_{1}$ and $N_{2}$ vary from $2 \sim 18$. At first, it can be seen in Figure 13 that the total network throughput when using AIMD+ either remains equal or higher than the case when using baseline. Secondly, throughput for the nodes in Zone 2 always remained higher for all the values of $N_{2}$. Thirdly, we can see that throughputs of Zone 1 and Zone 2 nodes coincide when approaching the case $N_{1}=N_{2}=10$, again indicating that AIMD+ is more fair than the baseline in which the convergence of throughputs of Zone 1 and Zone 2 nodes happen when $N_{1}=3$ and $N_{2}=17$. Finally, it can be seen that throughput for the nodes in Zone 1 when using AIMD+ is always lower than when using baseline. It shows that AIMD+ effectively controls Zone 1 node's access to the channel in order to maintain channel access fairness among the nodes of two Zones as well as the total network throughput. 


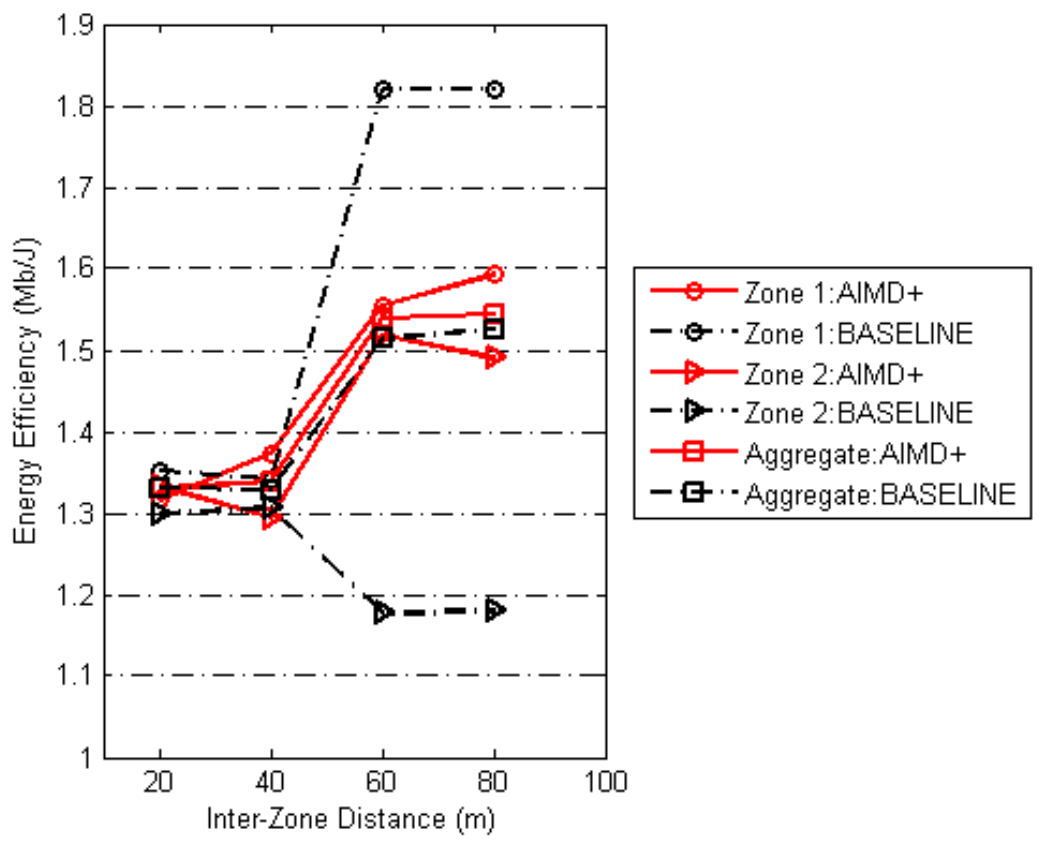

Figure 12. Comparison of AIMD+ and baseline with varying inter-zone distance.

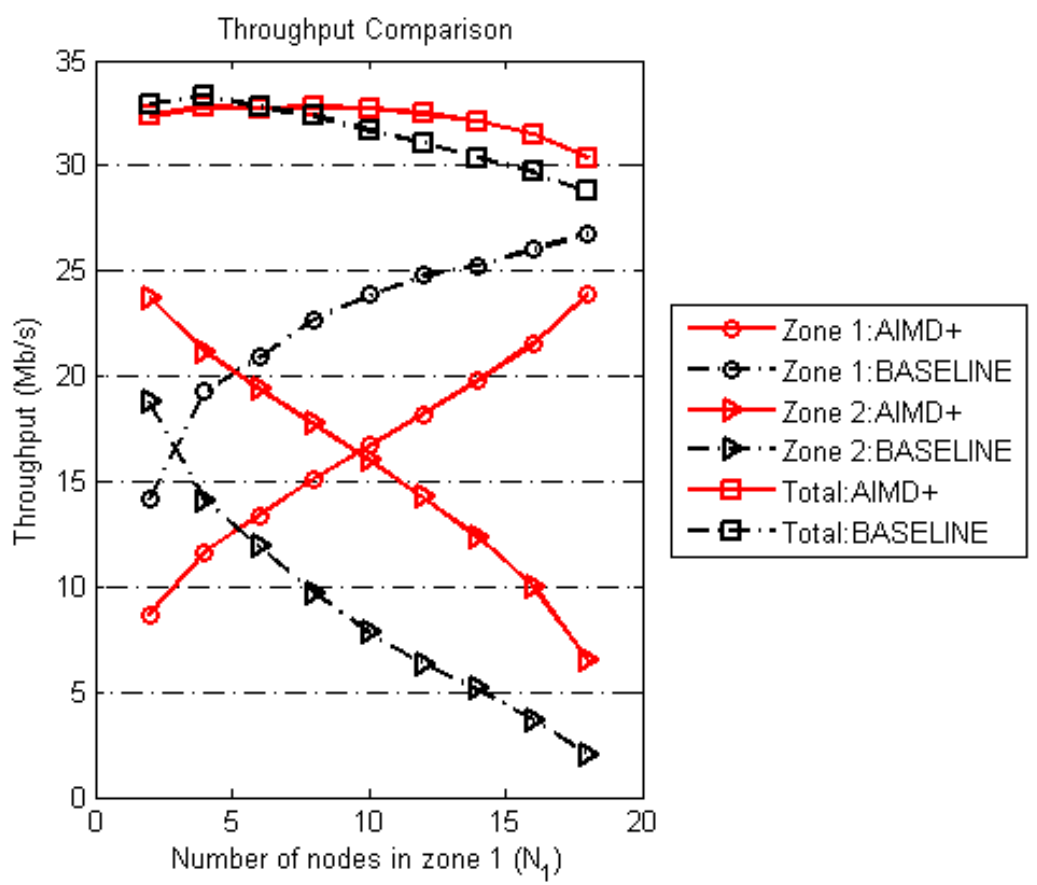

Figure 13. Throughput comparison of AIMD+ with baseline.

We, finally, compared the MAC delay incurred by packets to be successfully transmitted in the group of nodes in Zone 1 and Zone 2 using baseline and AIMD+ as shown in Figure 14. Similar to Figure 10, MAC delay in both zones was determined against the number of nodes in Zone 1 varying from two to 18 while the total number of nodes in network remained constant, i.e., $N_{\text {total }}=20$. At first, we see that when $N_{1}=2$ and $N_{2}=18$ delay for Zone 2 was only 13 ms while that for Zone 1 was around $2 \mathrm{~ms}$ when using the baseline, however, this delay for both zones increases gradually for both zones when $N_{1}=18$ and $N_{2}=2$. From this observation we can say that baseline is extremely unfair as it imposes unnecessary delay on the minimum number of Zone 2 nodes, i.e., when $N_{2}=2$. Another observation is that when $N_{1}=10$ and $N_{2}=10$ the delay incurred by Zone 2 is almost three times that 
of Zone 1 using baseline. In contrast to baseline, AIMD+ is extremely fair. For example, when the number of nodes in Zone 2 is reduced from $N_{2}=18$ to $N_{2}=2$ its MAC delay was reduced from $12 \mathrm{~ms}$ to $4.6 \mathrm{~ms}$ when using AIMD+. It clearly shows that Zone 2 delay was barely influenced by the number of nodes in Zone 1. Another useful observation that we can see from Figure 14 is that when there are equal number of nodes in each zone, $N_{1}=10$ and $N_{2}=10$ MAC delay incurred by each group of nodes is almost same showing that AIMD+ is fair in terms of delay among the two group of nodes.

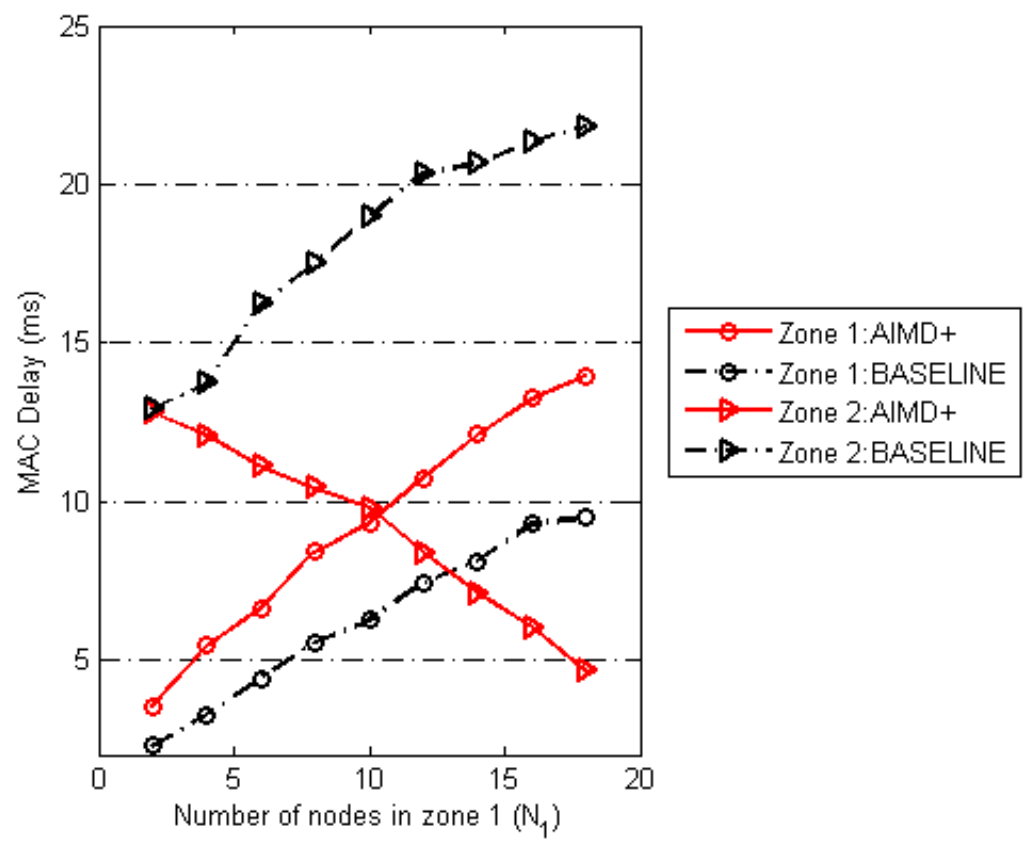

Figure 14. Medium access control (MAC) delay comparison of AIMD+ with baseline.

\section{Conclusions}

PHY capture effect has been shown to improve the throughput of wireless networks. However, its effect has not yet been explored in the energy conservation domain in WLAN. Our paper is the first effort in this regard. We at first presented an analytical model based on Markov chain that takes into account capture effect to calculate energy efficiency in the presence of various parameters, such as bit error rate and traffic load. The presented analytical model was also validated using simulation showing capture effect improves energy efficiency by $20 \%$ in a network of size 30 nodes. Secondly, we showed that although capture effect improves overall network energy efficiency but causes unfair energy consumption among different groups of nodes based on their respective distances from the AP. Thirdly, we proposed a remedy based on the combination of frame level adaptive transmission power control (ATXPR) and contention window adjustment (CWADJ) to overcome unfairness. Our proposed solution improves fairness by about $99 \%$ without degrading the overall aggregate energy efficiency and throughput.

Author Contributions: Conceptualization, B.K.; Data Curation, B.K.; Formal Analysis, B.K. and R.A.R.; Writing-Original Draft Preparation, B.K., R.A.R.; Writing-Review \& Editing, B.K., R.A.R. and B.-S.K.; Funding Acquisition, B.-S.K.

Funding: This work was supported by the Institute of Information and communications Technology Planning and Evaluation (IITP) grant funded by the Korea government(MSIT) (2018-0-01411, A Micro-Service IoTWare Framework Technology Development for Ultra small IoT Device).

Acknowledgments: We would like to thanks Byung-Seo Kim for his encouragement and sincere support during this research.

Conflicts of Interest: The authors declare that they have no any conflict of interest regarding this publication. 


\section{References}

1. Global e-Sustainability Initiative. SMART2020: Enabling the Low-Carbon Economy in the Information Age. Available online: http:/ / www.smart2020.org (accessed on 9 March 2019).

2. Carroll, Z.; Heiser, G. An analysis of power consumption in a smartphone. In Proceedings of the 2010 USENIX Annual Technical Conference, Boston, MA, USA, 23-25 June 2010.

3. IEEE 802.11 Working Group. Part 11: Wireless LAN Medium Access Control (MAC) and Physical Layer (PHY) Specifications; IEEE: Piscataway, NJ, USA, 2012.

4. Agrawal, P.; Kumar, A.; Kuri, J.; Panda, M.K.; Navda, V.; Ramjee, R.; Padmanabhani, V.N. Analytical models for energy consumption in infrastructure wlan stas carrying tcp traffic. In Proceedings of the 2010 Second International Conference on COMmunication Systems and NETworks (COMSNETS 2010), Bangalore, India, 5-9 January 2010.

5. He, Y.; Yuan, R.; Ma, X.; Li, J. The IEEE 802.11 Power Saving Mechanism: An Experimental Study. In Proceedings of the 2008 IEEE Wireless Communications and Networking Conference, Las Vegas, NV, USA, 31 March-3 April 2008.

6. Kochut, A.; Vasan, A.; Shankar, A.U.; Agrawala, A. Sniffing out the correct physical layer capture model in 802.11b. In Proceedings of the 12th IEEE International Conference on Network Protocols, Berlin, Germany, 8 October 2004.

7. Jung, D.; Kim, R.; Lim, H. Power-Saving Strategy for Balancing Energy and Delay Performance in WLANs. Comput. Commun. 2014, 50, 3-9. [CrossRef]

8. Agrawal, P.; Kumar, A.; Kuri, J.; Panda, M.K.; Navda, V.; Ramjee, R. OPSM-Opportunistic Power Save Mode for Infrastructure IEEE 802.11 WLAN. In Proceedings of the IEEE International Conference on Communications Workshops, Capetown, South Africa, 23-27 May 2010.

9. Qiao, D.; Choi, S.; Soomro, A.; Shin, K.G. Energy-efficient PCF operation of IEEE 802.11a WLANs via transmit power control. Comput. Netw. 2003, 42, 39-54. [CrossRef]

10. Tauber, M.; Bhatti, S.N. The effect of 802.11 psm on energy efficiency and performance during system activity. In Proceedings of the 2012 IEEE International Conference on Green Computing and Communications (GreenCom), Besancon, France, 20-23 November 2012; pp. 573-580.

11. Rozner, E.; Navda, V.; Ramjee, R.; Rayanchu, S. NAPman: Network-assisted power management for wifi devices. In Proceedings of the of ACM MobiSys, San Francisco, CA, USA, 15-18 June 2010.

12. Daneshgaran, F.; Laddomada, M.; Mesiti, F.; Mondin, M. Unsaturated Throughput analysis of ieee 802.11 in presence of non ideal transmission channel and capture effectsl. IEEE Trans. Wirel. Commun. 2008, 7, 1276-1286. [CrossRef]

13. Sutton, G.J.; Liu, R.P.; Collings, I.B. Modelling IEEE 802.11 DCF Heterogeneous Networks with Rayleigh Fading and Capture. IEEE Trans. Commun. 2013, 61, 3336-3348. [CrossRef]

14. Kumar, P.; Krishnan, A. Throughput Analysis of the IEEE 802.11 Distributed Coordination Function Considering Erroneous Channel and Capture Effects. Int. J. Autom. Comput. 2011, 8, 236-243. [CrossRef]

15. Nandagopal, T.; Kim, T.E.; Gao, X.; Bharghavan, V. Achieving MAC Layer Fairness in Wireless Packet Networks. In Proceedings of the 6th Annual International Conference on Mobile Computing and Networking, Boston, MA, USA, 6-11 August 2000; pp. 87-98.

16. Brown, T. X.; Gabow, H.N.; Zhang, Q. Maximum flow-life curve for a wireless ad hoc network. In Proceedings of the 2nd ACM International Symposium on Mobile Ad Hoc Networking and Computing, Long Beach, CA, USA, 4-5 October 2001; Volume 1, pp. 128-136.

17. Krishnamachari, B.; Ordonez, F. Analysis of energy-efficient fair routing in wireless sensor networks through non-linear optimization. In Proceedings of the IEEE 58th Vehicular Technology Conference, Orlando, FL, USA, 6-9 October 2003; Volume 5, pp. 2844-2848.

18. Mohajerzadeh, A.H.; Yaghmaee, M.H.; Eskandari, Z. Tree based energy efficient and congestion aware routing protocol for wireless sensor networks. In Proceedings of the 11th IEEE Singapore International Conference on Communication Systems, Guangzhou, China, 19-21 November 2008; Volume 1, pp. 1707-1711.

19. Safwati, A.; Hassanein, H.; Mouftah, H. Optimal cross-layer designs for energy-efficient wireless ad hoc and sensor networks. In Proceedings of the 2003 IEEE International Performance Computing and Communications Conference, Phoenix, AZ, USA, 9-11 April 2003; pp. 123-128. 
20. Shi, H.; Prasad, R.V.; Onur, E.; Niemegeers, I.G.M.M. Fairness in Wireless Networks-Issues, Measures and Challenges. IEEE Commun. Surv. Tutor. 2013, 16, 5-24.

21. Trifunovic, S.; Picu, A.; Hossmann, T.; Hummel, K. A. Slicing the Battery Pie: Fair and Efficient Energy Usage in Device-to-Device Communication via Role Switching. In Proceedings of the 8th ACM MobiCom Workshop on Challenged Networks, Miami, FL, USA, 30 September 2013; pp. 31-36.

22. Sodhro, A.H.; Li, Y.; Shah, M.A. Energy-efficient adaptive transmission power control for wireless body area networks. IET Commun. 2016, 10, 1-10. [CrossRef]

23. Sodhro, A.H.; Chen, L.; Sekhari, A.; Ouzrout, Y.; Wu, W. Energy efficiency comparison between data rate control and transmission power control algorithms for wireless body sensor networks. Int. J. Distrib. Sens. Netw. 2018, 14, 1-18. [CrossRef]

24. Su, S.L.; Tsai, Y.C.; Liao, H.C. Transmit Power Control Exploiting Capture Effect for WLANs. In Proceedings of the 7th IEEE International conference on Ubiquitous and Future Networks, Sapporo, Japa, 7-10 July 2015; pp. 634-638.

25. Lin, L.; Lin, X.; Shroff, N.B. Low-Complexity and Distributed Energy Minimization in Multi-Hop Wireless Networks. In Proceedings of the 26th IEEE International Conference on Computer Communications, Barcelona, Spain, 6-12 May 2007; pp. 1685-1693.

26. Kwon, S.; Shroff, N.B. Energy-Efficient Interference-Based Routing for Multi-hop Wireless Networks. In Proceedings of the 25TH IEEE International Conference on Computer Communications, Barcelona, Spain, 23-29 April 2006.

27. Lei, L.; Zhang, T. Saturation throughput analysis of IEEE 802.11 DCF with heterogeneous node transmit powers and capture effect. Int. J. Adhob Ubiquitous Comput. 2017, 26, 1-11. [CrossRef]

28. Jain, R.K.; Chiu, D.M.; Hawe, W.R. A Quantitative Measure of Fairness and Discrimination for Resource Allocation in Shared Computer Systems; DEC Research Report DEC-TR-301; Digital Equipment Corporation: Hudson, MA, USA, 1984.

29. Bianchi, G. Performance analysis of IEEE 802.11 distributed coordination function. IEEE J. Sel. Areas Commun. 2000, 18, 535-547. [CrossRef]

30. LaMaire, R.O.; Krishna A.; Zorzi, M. On the randomization of transmitter power levels to increase throughput in multiple access radio systems. Wirel. Netw. 1998, 4, 263-277. [CrossRef]

31. Hadzi-Velkov, Z.; Spasenovski, B. Capture effect with diversity in IEEE 802.11b DCF. In Proceedings of the IEEE 8th international Symposium on Computers and Communication, Kemer-Antalya, Turkey, 3 July 2003.

32. ns-2. Network Simulator. Available online: www.isi.edu/nsnam/ns (accessed on 9 March 2019) 